\title{
L- ve M-zayıf Kompakt Operatörlerin Sıra Yapısı Üzerine
}

\author{
Erdal BAYRAM ${ }^{*}$
}

Tekirdă̆ Namık Kemal Üniversitesi, Matematik Bölümü, Tekirdă̆

\begin{abstract}
$\ddot{O} \mathbf{z}$
Bu çalışmada Banach örgüleri arasında tanımlı L-zayıf ve M-zayıf kompakt operatörlerin sıra yapısı ile ilgili olarak regüler operatörler sınıfı içinde band ve KB-uzay olma koşullarına dair bazı sonuçlar verilmiştir.
\end{abstract}

Anahtar kelimeler: Banach örgüsü, L-zayıf kompakt operatör, M-zayıf kompakt operatör.

\section{On The Order Structure of L- and M-weakly Compact Operators}

\begin{abstract}
In this paper some conditions were given for the linear span of the positive L- and M-weakly compact operators defined between Banach lattices to be KB-space and band in the regular operators.
\end{abstract}

Keywords: Banach lattice, L-weakly compact operator, M-weakly compact operator.

\section{Giriş}

E Banach örgüsünün sınırlı bir alt kümesi A olmak üzere her bir $\left(x_{n}\right) \subset \operatorname{sol}(\mathrm{A})$ dik dizisi için $\left\|x_{n}\right\| \rightarrow$ 0 sağlanıyorsa A kümesine L-zayıf kompakt küme ve buna bağlı olarak $X$ Banach uzayının her bir sinırlı kümesini L-zayıf kompakt kümeye resmeden $T: X \rightarrow E$ sınırlı operatörüne L-zayıf kompakt operatör denir. Her bir sınırlı ve dik dizi $\left(x_{n}\right) \subset \mathrm{E}$ için $\left\|T x_{n}\right\| \rightarrow 0$ sağlanıyorsa $T: E \rightarrow X$ sınırlı operatörü de Mzayıf kompakt operatör olarak isimlendirilir. L-zayıf ve M-zayıf kompakt operatörler aynı zamanda zayıf kompakt operatörlerdir. Tanım ve görüntü kümelerinin AL- veya AM-uzay olma durumlarında ise zayıf kompakt operatörler ile çakışma durumları olabilmektedir. Zaten operatörlerin L- ve M- olarak isimlendirmeleri de buradan gelmektedir. Operatör sınıflarımız ilk olarak P. Meyer-Nieberg [1] tarafından tanımlanmış ve bir çok özelliği verilmiştir. Sonrasında ise literatürde oldukça fazla çalışma yer almıştır. Bu çalışmalar çoğunlukla operatörlerin birbiri arasındaki veya diğer operatör sınıfları ile olan ilişkileri hakkındadır. Buna karşın ilginçtir ki Riesz uzay yapılarına ilişkin sonuçlar elde edilmemiştir.

Banach örgüleri arasında tanımlı sınırlı operatör sınıflarının Riesz uzayı olabilme koşulları için baskınlık özelliği önemlidir. Baskınlık özelliği şu şekilde tanımlanabilir: $0 \leq S \leq T$ sağlandığında $S: E \rightarrow F$ operatörü ile $T: E \rightarrow F$ aynı sınıftandır. Kompakt, zayıf kompakt, Dunford-Pettis operatörleri gibi sınırlı operatörlerin bir çok alt sınıfı bu özelliğe sahip değildir ve sağlaması için bazı ek koşullar gerekir. Ancak L-zayıf ve M-zayıf kompakt operatörler hiçbir ek koşula gerek kalmadan baskınlık özelliğini sağlar. Bu nedenle bu operatör sınıfları bir çok sıra özelliklerine sahiptir. Fakat bu önemli özelliğe sahip olmalarına rağmen genel durumda Riesz uzayı oldukları söylenemez. Öyleki bir modüle sahip olamayan veya sahip olsa bile aynı operatör sınıfından olmayan regüler L-zayıf ve M-zayıf kompakt operatörler vardır. Örneğin Chen ve Wickstead [2] tarafından bu durumlara ilişkin sırasıyla $T: L^{2}[0,1] \rightarrow c\left(L^{2}[0,1]\right)$ ve $T: \ell_{1}\left(L^{2}[0,1]\right) \rightarrow \ell_{\infty}\left(L^{2}[0,1]\right)$ formunda örnekler verilmiştir. O halde $\mathcal{W}_{L, M}(E, F) \cap \mathcal{L}^{r}(E, F)$ ve $\mathcal{W}_{L, M}(E, F)$ Riesz uzayı olmak için fazlaca geniş operatör uzaylarıdır. Dolayısıyla bu sınıflardan daha dar olan M- ve L-zayıf kompakt pozitif operatörler ile üretilmiş sınıfları

"Sorumlu yazar: ebayram@nku.edu.tr

Geliş Tarihi: 13.06.2018, Kabul Tarihi: 27.11.2018 
düşünmek doğal olacaktır. Yakın zamanda Bayram ve Wickstead [3] bu sınıfların sıralı Banach uzay yapısına bağlı bir takım güzel sonuçlar elde etmişlerdir. Elde edilen sonuçların devamı olarak bu yayında L- ve M-zayıf kompakt pozitif operatörlerin ürettiği sınıfın KB-uzay ve band formunda olma durumları irdelenmiştir. Ayrıca $\mathcal{W}_{L, M}^{r}(E, F), \mathcal{W}_{L, M}(E, F) \cap L^{r}(E, F)$ ve $\mathcal{W}_{L, M}(E, F)$ sınıflarının çakıştıkları bazı durumlar verilmiştir.

\section{2. Önbilgiler ve Notasyon}

Banach örgüleri ve üzerlerinde tanımlanan operatörler hakkındaki temel tanım ve gösterimler için [4] ve [5] referans olarak alınmıştır. Aksi söylenmedikçe E ve F Banach örgüleri, X ve Y Banach uzayları olarak kabul edilecek, operatör kelimesi lineer ve sınırlı dönüşümler için kullanılacak ve bazı operatör sınıfları için aşağıdaki notasyonlar geçerli olacaktır.

$$
\begin{aligned}
& \mathscr{L}(X, Y)=\{T: X \rightarrow Y: T \text { lineer ve sürekli }\} \\
& \mathscr{L}^{+}(E, F)=\left\{T \in \mathscr{L}(E, F): T\left(E^{+}\right) \subseteq F^{+}\right\} \\
& \mathscr{L}^{r}(E, F)=\left\{T_{1}-T_{2}: T_{1}, T_{2} \in \mathscr{L}^{+}(E, F)\right\} \\
& \mathcal{W}_{L}(X, E)=\{T \in \mathscr{L}(X, E): T, L-\text { zayıf kompakt operatör }\} \\
& \mathcal{W}_{L}^{+}(E, F)=\mathcal{W}_{L}(E, F) \cap \mathscr{L}^{+}(E, F) \\
& \mathcal{W}_{L}^{r}(E, F)=\left\{T_{1}-T_{2}: T_{1}, T_{2} \in \mathcal{W}_{L}^{+}(E, F)\right\} \\
& \mathcal{W}_{M}(E, X)=\{T \in \mathscr{L}(\mathrm{E}, \mathrm{X}): T, M-\text { zayıf kompakt operatör }\} \\
& \mathcal{W}_{M}^{+}(E, F)=\mathcal{W}_{M}(E, F) \cap \mathscr{L}^{+}(E, F) \\
& \mathcal{W}_{M}^{r}(E, F)=\left\{T_{1}-T_{2}: T_{1}, T_{2} \in \mathcal{W}_{M}^{+}(E, F)\right\}
\end{aligned}
$$

Her iki operatör sınıfı için geçerli bir hususta $\mathcal{W}_{L, M}(E, F)$ kullanılacaktır.

$\mathscr{L}(E, F)$ genelde vektör örgüsü ve operatör normuna göre Banach uzayı olmak zorunda değildir. Fakat regüler norm olarak adlandırdığımız ve

$$
\|T\|_{r}=\inf \left\{\|S\|: S \in \mathscr{L}(E, F)_{+},|T x| \leq S|x|, \forall x \in E\right\}
$$

şeklinde tanımlı norm ile $\mathscr{L}^{r}(E, F)$ bir Banach uzayı olacaktır. Üstelik $F$ Dedekind tam olması durumunda ise $\left(\mathscr{L}(E, F),\|.\|_{r}\right)$ bir Banach örgüsüdür. Böylece $T \in \mathscr{L}(E, F)$ operatörü bir modüle sahiptir ve $\|T\|_{r}=\||T|\|$ sağlanır. Fakat $\mathscr{L}(E, F)$ 'nin her alt sinıfı bu regüler norma göre Banach örgüsü olmak zorunda değildir. Örneğin regüler kompakt ve regüler zayıf kompakt operatörler vektör örgüsü değillerdir.

$\mathcal{W}_{L, M}(E, F)$ zayıf kompakt operatörlerin bir alt sınıfı olmalarına rağmen kompakt operatörlerle mutlak bir ilişkileri yoktur. Yani genelde kompakt, L-zayıf kompakt ve M-zayıf kompakt operatörler farklı sınıflardır. Önemli bir diğer özellikleri ise dual operatörlerinde bir birlerine dönüşmeleridir. Yani, bir operatörün L-zayıf (veya M-zayıf) kompakt olması için gerekli ve yeterli koşul M-zayıf (veya Lzayıf) kompakt olmasıdır.

Yakın zamanda elde edilen $\mathcal{W}_{M, L}^{r}(E, F)$ 'nin sıralı Banach uzay yapısına ilişkin önemli iki teorem aşağıda verilmiştir.

Teorem 2.1 $\mathcal{W}_{L}^{r}(E, F)$ regüler norm ile Dedekind tam Banach örgüsüdür [3].

Teorem 2.2 F Dedekind tam ise $\mathcal{W}_{M}^{r}(E, F)$ regüler norm ile Dedekind tam Banach örgüsüdür [3].

$x_{\alpha} \downarrow 0$ olacak biçimdeki her $\operatorname{bir}\left(x_{\alpha}\right) \subset E$ için $\left\|x_{\alpha}\right\| \downarrow 0$ sağlanıyorsa $E$ sıra sürekli norma sahiptir denir. Örneğin standart normları ile $L^{1}[0,1], \ell_{1}, c_{0}$ sıra sürekli norma sahip olmalarına karşın 
$L^{\infty}[0,1], \ell_{\infty}, c$ sıra sürekli norma sahip değillerdir. $E$ Banach örgüsünün sıra sürekli kısmı olarak adlandırılan

$$
E^{a}=\{x \in E:[0,|x|] \text { içinde her monoton dizi yakınsak }\}
$$

kümesi sıra sürekli norma sahip maksimal idealdir. Örneğin $\ell_{\infty}{ }^{a}=c^{a}=c_{0}$ ve $\mu$ atomsuz bir ölçü olmak üzere $(\mathrm{L}(\mu))^{a}=\{0\}$ olur. $E^{a}$ ideali L-zayıf kompakt operatörler için önemli bir araçtır. Çünkü tüm L-zayıf kompakt kümeler $E^{a}$ tarafından kapsanacağından her $\mathrm{T} \in \mathcal{W}_{L}(X, E)$ için $T(X) \subset \mathrm{E}^{\mathrm{a}}$ sağlanır. Yani aslında $\mathcal{W}_{L}(X, E)=\mathcal{W}_{L}\left(X, E^{a}\right)$ olur.

E Banach örgüsündeki her bir norm sınırlı monoton dizi yakınsak ise E bir KB-uzaydır (Kantorovic-Banach) denir. KB-uzayları sıra sürekli norma sahip uzaylardır fakat bunun tersi doğru değildir. Örneğin $c_{0}$ sıra sürekli norma sahip olmasına karşın KB-uzay değildir. Banach örgülerinin önemli alt kümelerinden biri de bandlerdir. Bir Riesz uzayının sıra kapalı ideallerine band denir. Dolayısıyla $\mathrm{A} \subset E$ idealinin band olabilmesi için gerekli ve yeterli koşul A 'nın $E$ içinde supremuma sahip her alt kümesinin supremumunun $\mathrm{A}$ 'ya ait olmasıdır. Örneğin $c_{0}, \ell_{\infty}$ içinde bir ideal olmasına karşın band değildir.

\section{3. $\mathcal{W}_{\mathrm{L}}(E, F)$ ve $\mathcal{W}_{\mathrm{M}}(E, F)$ 'nin Riesz Uzay Yapısı} olarak

L- ve M-zayıf kompakt operatörler $\mathscr{L}(E, F)$ içinde kapalı vektör uzayı olduklarından genel

$$
\mathcal{W}_{L, M}^{r}(E, F) \subseteq \mathcal{W}_{L, M}(E, F) \cap L^{r}(E, F) \subseteq \mathcal{W}_{L, M}(E, F)
$$

kapsamaları sağlanır. Diğer taraftan yukarıda bahsedilen örnekler göstermektedir ki kapsamalar öz olabilir. Aşağıdaki basit sonuç ise bu kapsamaların eşit olabileceği bir durumu belirtir.

Önerme 3.1 Her bir $T \in \mathcal{W}_{L}(E, F)$ operatörünün modülü $|T|$ var ve $|T| \in \mathcal{W}_{L}(E, F)$ olmas1 için gerek ve yeter koşul aşağıdaki eşitliklerin sağlanmasıdır.

$$
\mathcal{W}_{L}^{r}(E, F)=\mathcal{W}_{L}(E, F) \cap \mathscr{L}^{r}(E, F)=\mathcal{W}_{L}(E, F) .
$$

İspat: Kabul edelim ki her bir $T \in \mathcal{W}_{L}(E, F)$ için $|T|$ var ve L-zayıf kompakt olsun. $T \in \mathcal{W}_{L}(E, F) \cap$ $\mathscr{L}^{P}(E, F)$ ise $0 \leq T^{+}, T^{-} \leq|T|$ sağlandı̆̆ından baskınlık özelliği gereği $T^{+}, T^{-} \in \mathcal{W}_{L}(E, F)_{+}$olur. Dolayısıyla $T=T^{+}-T^{-} \in \mathcal{W}_{L}^{r}(E, F)$ yazılabileceğinden $\mathcal{W}_{L}^{r}(E, F)=\mathcal{W}_{L}(E, F) \cap \mathscr{L}^{r}(E, F)$ sağlanır. Diğer yandan her bir $T \in \mathcal{W}_{L}(E, F)$ için $|T| \in \mathcal{W}_{L}(E, F)$ ve $\mathcal{W}_{L}(E, F)$ kapalı vektör uzayı olduğundan $T=|T|-(|T|-T) \in \mathcal{W}_{L}(E, F) \cap \mathscr{L}(E, F) \quad$ yazilabilmesi $\quad \mathcal{W}_{L}(E, F) \subset \mathcal{W}_{L}(E, F) \cap \mathscr{L}^{r}(E, F)=$ $\mathcal{W}_{L}^{r}(E, F)$ kapsamasını verir ki bu da istenen eşitliğin doğru olduğunu gösterir. Tersine bu eşitliğin doğru olduğunu kabul ettiğimizde Teorem 2.1 ile $\mathcal{W}_{L}^{r}(E, F)$ 'nin Dedekind tam Banach örgüsü olduğunun ispatı $\mathcal{W}_{L}(E, F)$ 'nin de Banach örgüsü olmasını gerektirir. Yani her bir $T \in \mathcal{W}_{L}(E, F)$ için $|T| \in \mathcal{W}_{L}(E, F)$ olur.

Benzer sonucu M-zayıf kompakt operatörler için ele aldığımızda $F$ Banach örgüsünün sıra tam olma koşulunun ek olarak var olması gerekliliği ortaya çıkar. Bunun sebebi ispatta kullanılan L-zayıf kompakt operatörlerin Banach örgüsü formunda olmasının her zaman sağlanmasına karşın bu durum M-zayıf kompakt operatörler için doğru değildir. Çünkü her bir L-zayıf kompakt operatör $F$ Banach örgüsünün zaten sıra tam olan sıra sürekli kısmı $F^{\mathrm{a}}$ 'da değer alır.

Önerme 3.2 $F$ Dedekind tam olsun. Bu durumda $T \in \mathcal{W}_{M}(E, F)$ operatörünün modülü $|T|$ var ve $|T| \in$ $\mathcal{W}_{M}(E, F)$ olması için gerek ve yeter koşul aşağıdaki eşitliklerin sağlanmasıdır.

$$
\mathcal{W}_{M}^{r}(E, F)=\mathcal{W}_{M}(E, F) \cap \mathscr{L}^{r}(E, F)=\mathcal{W}_{M}(E, F) .
$$

İspat: $F$ Dedekind tam ise $\mathcal{W}_{M}(E, F)$ Dedekind tam Banach örgüsüdür. Geriye kalan ispat Önerme 3.1 'in ispatı ile aynıdır.

Bu durumda aşağıdaki iki sonuç kolaylıkla görülür. 
Sonuç 3.3 $\mathcal{W}_{L}^{r}(E, F)=\mathcal{W}_{L}(E, F) \cap \mathscr{L}^{r}(E, F)=\mathcal{W}_{L}(E, F)$ eşitlikleri aşağıdaki şartlardan herhangi biri var ise sağlanır.

1. E bir AL-uzayına örgü izomorftur.

2. $F$ bir AM-uzayıdır.

İspat: [2] 'deki Theorem 2.4 ve 2.9 ile Önerme 3.1 'den ispat açıktır.

Sonuç 3.4 $\mathcal{W}_{M}^{r}(E, F)=\mathcal{W}_{M}(E, F) \cap \mathscr{L}^{r}(E, F)=\mathcal{W}_{M}(E, F)$ eşitlikleri aşağıdaki şartlardan herhangi biri var ise sağlanır.

1. $F$ güçlü birime sahip ve sıra tamdır.

2. E bir AL-uzayıdır.

İspat: [2] 'deki Theorem 2.5 ve 2.6 ile Önerme 3.2 den ispat açıktır.

\section{4. $\mathcal{W}_{L}^{r}(E, F)$ ve $\mathcal{W}_{M}^{r}(E, F)$ Ne Zaman KB-uzayı Veya Banddir?}

$S \in \mathcal{W}_{L}^{r}(E, F)$ ve $T \in L^{r}(E, F)$ için $|T|$ var olsun ve $|T| \leq|S|$ sağlansın. Bu durumda $\operatorname{sol}(|T|) \subseteq$ $\operatorname{sol}(|S|)$ kapsaması sağlanacağından $|T| \in \mathcal{W}_{L}(E, F)$ ve dolayısıyla $T \in \mathcal{W}_{L}^{r}(E, F)$ olur. Benzer olarak $F$ Dedekind tam ise, $S \in \mathcal{W}_{M}^{r}(E, F)$ ve $T \in \mathscr{L}^{r}(E, F)$ için $|T|$ var ve $|T| \leq|S|$ sağlanıyorsa $|T| \in$ $\mathcal{W}_{M}(E, F)$ ve dolayisiyla $T \in \mathcal{W}_{M}^{r}(E, F)$ olur. Buradan $\mathcal{W}_{L}^{r}(E, F)$ ve $\mathrm{F}$ sira tam ise $\mathcal{W}_{M}^{r}(E, F)$ operatör sınıflarının $\mathscr{\mathscr { L }}(E, F)$ içinde birer sıra ideal oldukları görülür. Ancak bu operatör sınıfları $\mathscr{L}(E, F)$ içinde band olmak zorunda değildir.

Örneğin, [6] 'da her bir $\forall n \in \mathrm{N}$ için tanımlanan $T_{n}: c_{0} \rightarrow c_{0}, T_{n}\left(\lambda_{k}\right)=\left(\lambda_{1}, \lambda_{2}, \ldots, \lambda_{n}, 0,0, \ldots\right)$ pozitif kompakt operatörleri $c_{0}$ and $\left(c_{0}\right)^{\prime}$ sıra sürekli norma sahip olduğundan aynı zamanda L-zayıf ve M-zayıf kompaktır. Üstelik $I$ özdeşlik dönüşümü olmak üzere $\mathscr{L}^{r}\left(c_{0}\right)$ 'de $T_{n} \uparrow I$ sağlanır. Fakat $I \notin$ $\mathcal{W}_{L}^{r}\left(c_{0}\right)$ ve $I \notin \mathcal{W}_{M}^{r}\left(c_{0}\right)$ olur. Yani $\mathcal{W}_{L, M}^{r}\left(c_{0}\right), \mathscr{L}^{r}\left(c_{0}\right)$ içinde band değildir.

$E^{\prime}$ pozitif Schur özelliğine sahip ve $F$ bir KB-uzayı veya $F$ pozitif Schur özelliğine sahip ve $E^{\prime}$ sıra sürekli norma sahip ise $r$-kompakt operatörler $\mathscr{F}(E, F)$ ile pozitif kompakt operatörlerin ürettiği sınıf $\mathcal{K}^{r}(E, F)$ 'nin $\mathscr{L}^{r}(E, F)$ içinde band oldukları ispatlanmıştır ([6], Theorem 3.8 and 3.9). Bu ispatlarda $F$ sira sürekli norma sahip olduğunda operatör normunun $[0, T]$ üzerinde sira sürekli olmas1 için gerek ve yeter şartın $T$ operatörünün M-zayıf kompakt olması gerekliliği kullanılmıştır. Ancak aynı kabuller altında $\mathscr{L}(E, F)=\mathcal{W}_{L}^{r}(E, F)=\mathcal{W}_{M}^{r}(E, F)$ eşitliği sağlanacağından $\mathcal{W}_{L}^{r}(E, F)$ ve $\mathcal{W}_{M}^{r}(E, F)$ $\operatorname{nin} \mathscr{L}^{r}(E, F)$ içinde band olduğu barizdir ([2], Theorem 3.6, 3.7, 3.8, 3.9). Benzer olarak $1 \leq p<q \leq$ $\infty$ olmak üzere $E q$-alt toplamsal norma ve $F p$-süper toplamsal norma sahip ise her regüler operatör Lzayıf ve M-zayıf kompakttır ([5], Proposition 3.6.20).

Aşağıdaki önerme [6], Theorem 3.7 'ye benzer olarak $\mathcal{W}_{L}^{r}(E, F)$ ve $\mathcal{W}_{M}^{r}(E, F)$ 'nin band olduğu bir durumu belirtmektedir.

Teorem 4.1 $E$ güçlü sıra birime sahip AM-uzayı ve $F$ sıra sürekli norma sahip ise $\mathcal{W}_{L}^{r}(E, F)$ ve $\mathcal{W}_{M}^{r}(E, F), \mathscr{L}^{r}(E, F)$ içinde banddir.

İspat: [3] 'de Theorem 2.3 (sırasıyla Theorem 2.4) gereği $\mathcal{W}_{L}^{r}(E, F)$ (sırasıly $\mathcal{W}_{M}^{r}(E, F)$ ) üzerindeki regüler norm sıra süreklidir. Kabul edelimki $\mathscr{L}^{r}(E, F)$ içinde $0 \leq T_{\alpha} \in \mathcal{W}_{L}^{r}(E, F)$ (sırasıly $\mathcal{W}_{M}^{r}(E, F)$ ), için $T_{\alpha} \uparrow T$ sağlansın. Bu durumda $F$ (sırasıyla $E^{\prime}$ ) sıra sürekli norma sahip olduğundan güçlü operatör topolojisine göre $T_{\alpha} \rightarrow T$ sağlanır. Dolayısıyla $E$ 'nin güçlü sıra birimi $e$ için

$$
\left\|T-T_{\alpha}\right\|_{r}=\left\|\left|T-T_{\alpha}\right|\right\|=\left\|T-T_{\alpha}\right\|=\left\|T e-T_{\alpha} e\right\| \rightarrow 0
$$

olur. Bu durumda $\mathcal{W}_{L}^{r}(E, F)$ (sırasıly $\left.\mathcal{W}_{M}^{r}(E, F)\right) \mathscr{L}^{r}(E, F)$ içindeki kapalılığından $T \in \mathcal{W}_{L}^{r}(E, F)$ (sırasıyla $T \in \mathcal{W}_{M}^{r}(E, F)$ ) olduğu görülür.

Aşağıdaki lemma, regüler norma göre $\mathcal{W}_{L}^{r}(E, F)$ ve $\mathcal{W}_{M}^{r}(E, F)$ operatör sınıflarının $K B$-uzayı olduğu bir durumu vermek için kullanılacaktır. $f \in E^{\prime}$ ve $y \in \mathrm{F}$ için $f \otimes y: E \rightarrow F$ dönüşümü

$$
\mathrm{x} \rightarrow(f \otimes y)(x)=f(x) y
$$


olarak tanımlanmıştır. Buna göre açıktır ki; $0 \neq y \in F^{a}$ ve $f \in E^{\prime}$ için $f \otimes y \in \mathcal{W}_{L}(E, F)$ ve benzer olarak $0 \neq x \in E$ ve $f \in\left(E^{\prime}\right)^{a}$ için $f \otimes y \in \mathcal{W}_{M}(E, F)$ olacaktır. Diğer taraftan $\left(E^{\prime}\right)^{a} \neq\{0\} \neq F^{a}$ şartı, kullandığımız operatör sınıflarının özdeş olarak sıfır uzayı olmaması istendiğindendir. Çünkü $F^{a}=\{0\}$ (sırasıyla $\left(E^{\prime}\right)^{a}=\{0\}$ ) olması için gerek ve yeterli koşul $\mathcal{W}_{L}(E, F)=\{0\}$ (sırasıyla $\left.\mathcal{W}_{M}(E, F)=\{0\}\right)$ olmasidir.

Lemma 4.1 $\left(E^{\prime}\right)^{a} \neq\{0\} \neq F^{a}$ olmak üzere aşağıdakiler sağlanır.

1. $E^{\prime}, \mathcal{W}_{L}^{r}(E, F)$ içine pozitif olarak gömülebilir.

2. $E^{\prime}$ sira sürekli norma sahip ise $E^{\prime}, \mathcal{W}_{M}^{r}(E, F)$ içine pozitif olarak gömülebilir.

3. $F, \mathcal{W}_{M}^{r}(E, F)$ içine pozitif olarak gömülebilir.

4. $F$ sıra sürekli norma sahip ise $F, \mathcal{W}_{L}^{r}(E, F)$ içine pozitif olarak gömülebilir.

İspat: $\|y\|=1$ olacak biçimde $y \in F_{+}^{a}$ seçelim. Buna göre

$$
\phi: E^{\prime} \rightarrow \mathcal{W}_{L}^{r}(E, F), \phi(f)=f \otimes y
$$

operatörü pozitiftir ve $\|\phi(f)\|=\|f\|$ sağlandığından $\phi$ bir izometridir. Yani bir örgü gömme dönüşümüdür. Dolayısıyla (1) sağlanır. Benzer olarak $\|f\|=1$ olacak biçimde $f \in\left(E^{\prime}\right)_{+}^{a}$ seçersek

$$
\psi: F \rightarrow \mathcal{W}_{M}^{r}(E, F), \phi(y)=f \otimes y
$$

operatörü pozitiftir ve $\|\phi(y)\|=\|y\|$ sağlanacağından (3) görülür. Diğer taraftan $E^{\prime}$ (sırasıyla $F$ ) sıra sürekli norma sahip ise $\mathcal{W}_{L}^{r}(E, F) \subseteq \mathcal{W}_{M}^{r}(E, F)$ (sirasiyla $\mathcal{W}_{M}^{r}(E, F) \subseteq \mathcal{W}_{L}^{r}(E, F)$ ) sağlanacağından (2) ve (4) açıktır.

Teorem 4.2 $\left(E^{\prime}\right)^{a} \neq\{0\} \neq F^{a}$ ise aşağıdaki önermeler birbirine denktir.

1. $E^{\prime}$ ve $F, K B$-uzayları ise $\mathcal{W}_{L}^{r}(E, F), \mathscr{L}^{r}(E, F)$ içinde banddir.

2. $F$ sıra sürekli norma sahip ve $\mathcal{W}_{L}^{r}(E, F)$ regüler norma göre $K B$-uzayıdır.

İspat: $(1 \Rightarrow 2) F$ ve $E^{\prime}$ sıra sürekli norma sahip olduğundan [3] 'de Theorem 3.1 gereği açıktır ki $\left(\mathcal{W}_{L}^{r}(E, F),\|\cdot\|_{r}\right)$ sira sürekli norma sahip Banach örgüsüdür. Kabul edelim ki $\left(T_{n}\right) \subset \mathcal{W}_{L}^{r}(E, F)_{+}$ sınırlı artan bir dizi olsun. Dolayısıyla her bir $x \in E_{+}$için $\left(T_{n} x\right)$ sınırlı artan bir dizidir ve $F$ 'in $K B$ uzay olmasından dolayı yakınsaktır. Böylece güçlü operatör topolojiye göre $T_{n} \rightarrow T$ olacak biçimde $0 \leq$ $T \in \mathscr{L}(E, F)$ operatörü tanımlanabilir. Yani $\mathscr{L}^{r}(E, F)$ içinde $T_{n} \uparrow T$ sağlanır. O halde kabulümüz gereği $\mathcal{W}_{L}^{r}(E, F)$ band olduğundan $T \in \mathcal{W}_{L}^{r}(E, F)$ olur. Son olarak regüler normun sira sürekliliğinden $\left\|T_{n}-T\right\|_{r} \rightarrow 0$ elde edilir.

$(2 \Rightarrow 1)$ Kabul edelim ki $F$ sira sürekli norma sahip ve regüler norma göre $\mathcal{W}_{L}^{r}(E, F) K B$-uzay olsun. [3] 'de Teorem 2.3 gereği $E^{\prime}$ sira sürekli norma sahiptir ve dolayısıyla bir $K B$-uzayıdır ([5], Theorem 2.4.14). Eğer $F$ bir $K B$-uzayı olmasayd $c_{0}$ dizi uzayına izomorfik bir alt örgüye sahip olacakt1 ([5], Theorem 2.4.12) ve dolayısıyla Lemma 4.1 gereği $\mathcal{W}_{L}^{r}(E, F)$ böyle bir alt örgüye sahip olacaktı. $\mathrm{Bu}$ ise $\mathcal{W}_{L}^{r}(E, F)$ nin regüler norma göre $K B$-uzay olmasına çelişkidir ([5], Theorem 2.4.12). Diğer taraftan $0 \leq T_{\alpha} \in \mathcal{W}_{L}^{r}(E, F)$ için $\mathscr{L}(E, F)$ içinde $T_{\alpha} \uparrow T$ sağlanıyorsa $\left\|T_{\alpha}\right\| \leq\|T\|$ olur. Yani $\left(T_{\alpha}\right)$, $\mathcal{W}_{L}^{r}(E, F)$ içinde norm sinırlı ve yukarı yönlendirilmiştir. $\mathcal{W}_{L}^{r}(E, F), K B$-uzay olduğundan $\left\|T_{\alpha}-S\right\|_{r} \rightarrow 0$ olacak biçimde $S \in \mathcal{W}_{L}^{r}(E, F)$ operatörü vardır. Dolayısıyla $S=\sup T_{\alpha}=T \in$ $\mathcal{W}_{L}^{r}(E, F)$ olacağından $\mathcal{W}_{L}^{r}(E, F)$ 'nin $\mathscr{L}^{r}(E, F)$ içinde band olduğu görülür.

Dual olarak M-zayıf kompakt operatörler için aşağıdaki benzer sonuç elde edilir.

Teorem $4.3\left(E^{\prime}\right)^{a} \neq\{0\} \neq F^{a}$ olmak üzere aşağıdaki önermeler birbirine denktir.

1. $F$ bir $K B$-uzayı ve $\mathcal{W}_{M}^{r}(E, F), \mathscr{L}^{r}(E, F)$ içinde banddir.

2. $\mathcal{W}_{M}^{r}(E, F)$ regüler norma göre $K B$-uzayıdır.

İspat: [3] 'de Theorem 2.4 gereği $F$ sira sürekli norma sahip olduğundan $\left(\mathcal{W}_{M}^{r}(E, F),\|.\|_{r}\right)$ sira sürekli norma sahip Banach örgüsüdür. Geriye kalan ispat Teorem 4.2 'in ispatına benzerdir. 


\section{Teşekkür}

Konu ile ilgili yararlı ve yapıcı yorumlarından dolayı A.W. Wickstead'a çok teşekkür ederim.

\section{Kaynaklar}

[1] Meyer-Nieberg P. 1974. Uber klassen schwach kompakter operatoren in Banachverbanden, Mathematische Zeitschrift, 138 (2): 145-159.

[2] Chen Z.L., Wickstead A.W. 1999. L-weakly and M-weakly compact operators, Indagationes Mathematicae, 10 (3): 321-336.

[3] Bayram E., Wickstead A.W. 2017. Banach lattices of L-weakly and M-weakly compact operators, Archiv der Mathematik (Basel), 108: 293-299.

[4] Aliprantis C.D., Burkinshaw O. 1985. Positive Operators, Academic Press, 367s. Orlando.

[5] Meyer-Nieberg P. 1991, Banach Lattices, Springer-Verlag, 395s. Berlin.

[6] Chen Z.L., Wickstead A.W. 2007. The Order Properties of r-compact Operators on Banach Lattices, Acta Mathematica Sinica, English Series, 23 (3): 457-466. 